\title{
Highly Miniaturized 1-4 On-Chip Power Divider/Combiner Circuit on Silicon Substrate for Application to Long Distance Wireless Power Transmission
}

\author{
Jeong-Gab Ju, Jang-Hyeon Jeong, Young-Bae Park, Bo-Ra Jung, Eui-Hoon Jang, Suk-Youb Kang, \\ and Young Yun
}

\begin{abstract}
In this paper, for application to a long distance wireless power transmission, a highly miniaturized 1-4 power divider/combiner employing periodic structure was fabricated on semiconducting silicon substrate. The 1-4 divider/combiner showed good RF performances from 1 to $25 \mathrm{GHz}$, and its size was $1.08 \times 0.63 \mathrm{~mm}^{2}$, which is $0.14 \%$ of the size of the one fabricated on PCB.
\end{abstract} AMP

Index Terms-Coplanar waveguide, PAGS, Silicon, RFIC,

\section{INTRODUCTION}

Wireless energy or power transfer is the transmission of electrical energy from a power source to a power consumer without interconnecting wires [1]-[4]. Wireless transmission is useful in cases where interconnecting wires are inconvenient, hazardous, or impossible. The most common form of wireless power transmission is carried out using electromagnetic /inductive coupling followed by resonant inductive coupling. However, inductive/electromagnetic coupling method is near field over distances comparable to a few times the diameter of the device or devices approaching one quarter of the wavelength used [1]. For a long distance wireless power transmission, wireless power transmission employing microwave is a promising candidate. Experiments in the tens of kilowatts have been performed by several groups[1]-[4]. These methods achieve distances on the order of a kilometer. Main applications of wireless power transmission employing microwave are shown in Fig. 1.

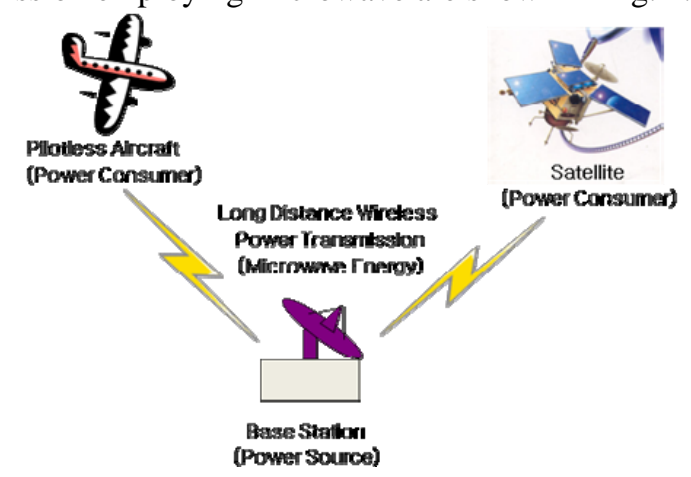

Fig. 1. Main applications of wireless power transmission employing microwave.
Base station transmits the power needed for the operation of pilotless aircraft and satellite. In this case, a long distance wireless power transmission is required to provide the operation power for the aircraft and satellite. The long distance wireless power transmission system is shown in Fig. 2. Power source provide microwave power using oscillator for wireless transmission. The microwave power is amplified by power amplifier, and amplified microwave power is transmitted to the power consumer.

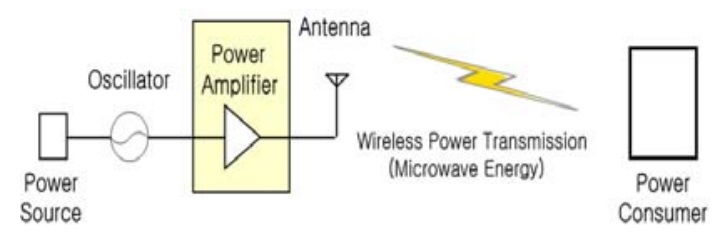

Fig. 2. The long distance wireless power transmission system.

In the above long distance wireless power transmission system, the power amplifier is a key device, because it amplifies the transmitted microwave power so that the power consumer operates well without additional power supply. Especially, a microwave power level of $1 \mathrm{~W}-1 \mathrm{~kW}$ is required for application to the long distance wireless power transmission for aircraft and satellite. In this case, the size of power amplifier is highly increased, and it occupies most of the area of the system. Therefore, the main issue of the long distance wireless power transmission is to reduce the size of the power amplifier. Especially, the size of the power divider/combiner should be highly reduced for a miniaturization of power amplifier, because the power divider/combiner occupies most of the area of the power amplifier [5]-[13]. In conventional system, the power divider/combiner was fabricated outside of semiconducting IC (Integrated Circuit) due to its large size.

In this work, to reduce the size of the power amplifier, highly miniaturized 1-4 power divider/combiner employing periodic structure was fabricated on semiconducting silicon substrate. The 1-4 divider/combiner showed good RF performances from 1 to $25 \mathrm{GHz}$ and its size was $1.08 \times 0.63$ $\mathrm{mm}^{2}$, which is $0.14 \%$ of the size of the one fabricated on PCB (Printed Circuit Board). This paper was selected from ICIIE (International Conference on Information and Industrial Electronics) 2011, and republished.

\section{Structure OF 1-4 POWER Divider/COMBINER}

Fig. 3 shows a schematic diagram of the power amplifier for a long distance power transmission. As shown in this 
figure, several amplifiers are connected in parallel using power divider/combiner in order to extract a high microwave power through power coupling. The size of the power combiner /divider should be highly reduced for a miniaturization of power amplifier, because the power divider/combiner occupies most of the area of the power amplifier [5]-[13]. In conventional system, the power divider/combiner was fabricated outside of semiconducting IC due to its large size. Fig. 4 shows the conventional X-band 1-4 divider/combiner fabricated on PCB. For, the power divider operation, the input microwave signal is applied to port 1 , and it is split into four output signals from port 2 - 5 . For the power combiner operation, the four input microwave signal are applied to port 2-5, and it is coupled at port 1 . However, the power divider on PCB occupies a very large area. The size of the X-band power divider/combiner is $25 \mathrm{X}$ $20 \mathrm{~mm}^{2}$, which increases the module size of the power amplifier.

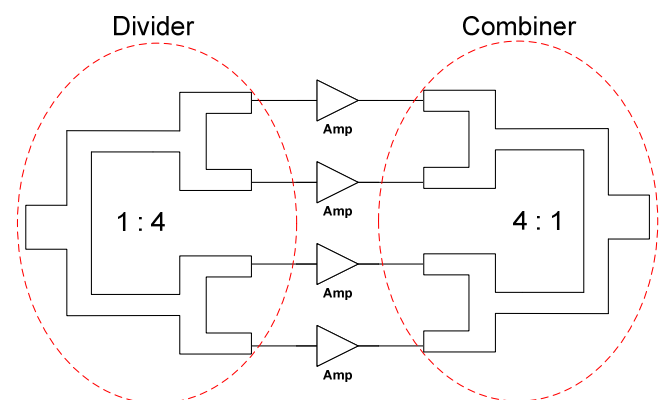

Fig. 3. The schematic diagram of power amplifier for a long distance power transmission.

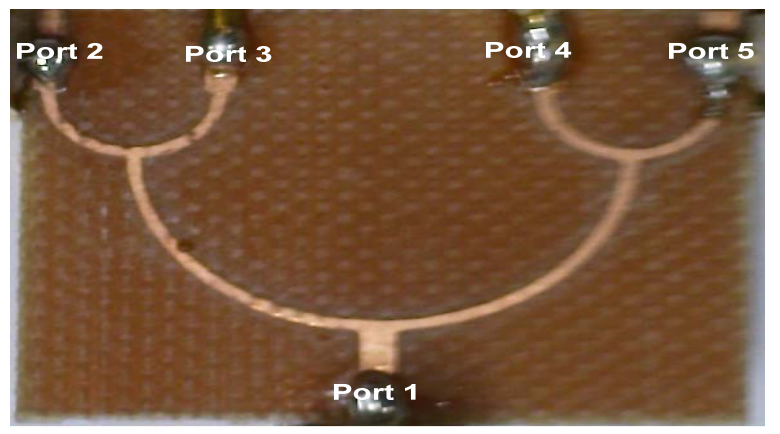

Fig. 4. Conventional 1-4 divider/combiner on PCB

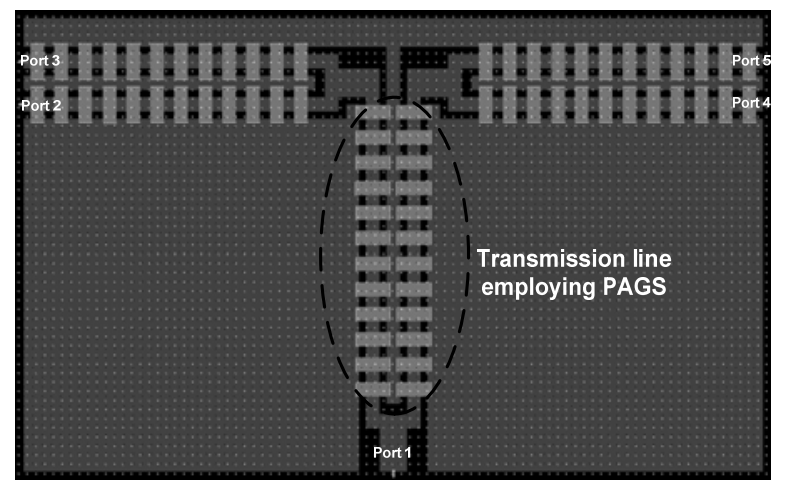

Fig. 5. A layout of the fabricated 1-4 power divider/combiner employing PAGS on silicon substrate

In this work, to reduce the size of the power amplifier, highly miniaturized 1-4 power divider/combiner employing periodic structure was fabricated on semiconducting silicon substrate. Fig. 5 shows a structure of the 1 to 4 power divider/combiner employing PAGS (Periodically Arrayed
Ground Strip) structure [14], [15] on silicon substrate. As shown in this figure, the power divider/combiner consists of the transmission line employing PAGS. Fig. 6 shows the PAGS structure [14], [15]. According to our previous report [14], [15], the transmission line employing PAGS showed slow-wave characteristics, and the wavelength of the transmission line employing PAGS was reduced to $60 \sim 65 \%$ of conventional one, which enables a miniaturization. Its size was $1.08 \times 0.63 \mathrm{~mm}^{2}$, which is $0.14 \%$ of the size of the conventional power divider/combiner fabricated on PCB (The size of the conventional power divider/combiner fabricated on PCB is $25 \mathrm{X} 20 \mathrm{~mm}^{2}$.)
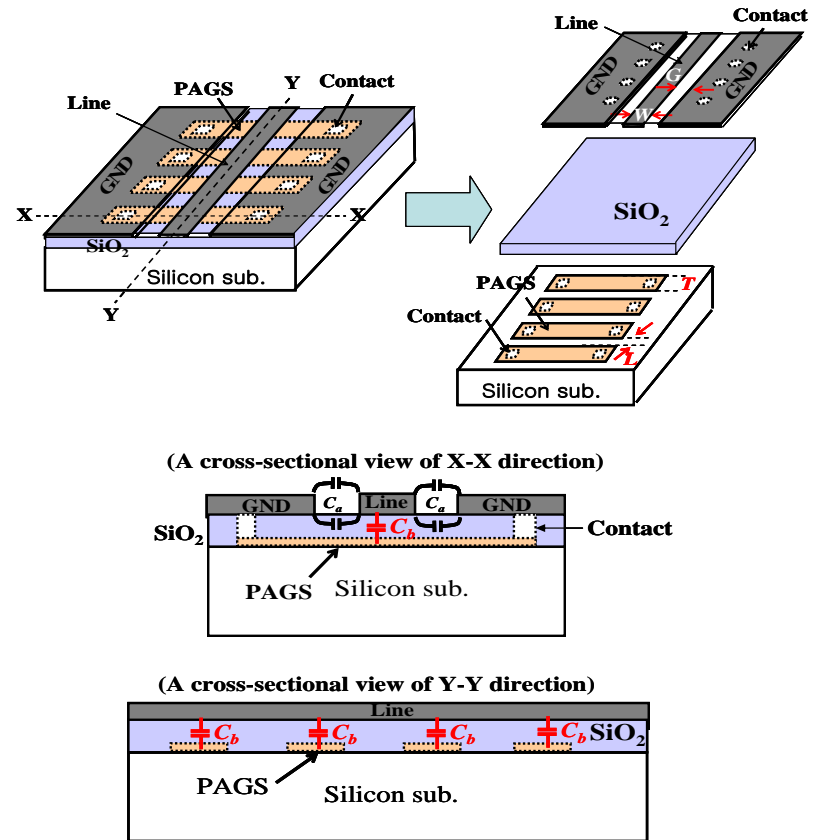

Fig. 6. Structure of the transmission line employing PAGS structure [14]

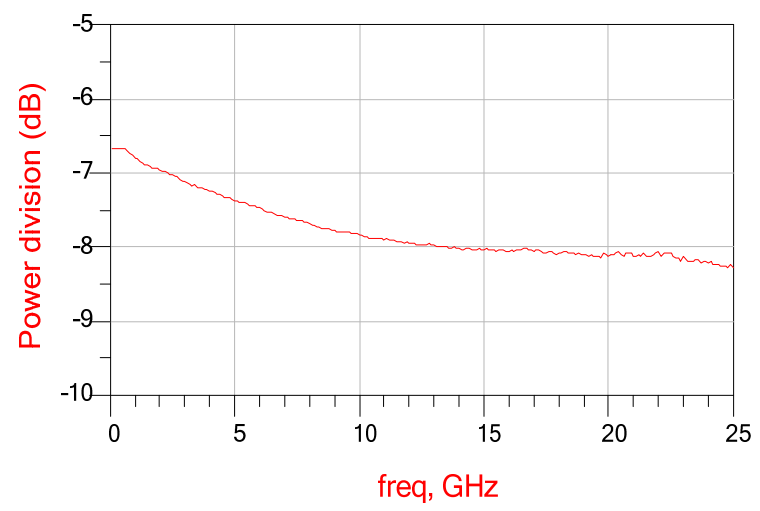

Fig. 7. Measured measured power division characteristics of the 1-4 power divider/combiner employing PAGS

\section{Performance OF 1-4 Power DiVIDER/COMBINER}

Actually, we fabricated 1-2 power divider/combiner on silicon substrate, and measured its RF characteristics. We extracted the RF performances of the 1-4 power divider /combiner from the above measured data. Fig. 7 and 8 show measured power division and isolation characteristics of the 1-4 power divider/combiner employing PAGS. We can observe good power division characteristic from 1 to $25 \mathrm{GHz}$. Concretely, power division exhibits a value of $-7.5 \mathrm{~dB}$ at 10 $\mathrm{GHz}$. In a frequency range of 1 to $25 \mathrm{GHz}$, show a value of $-7.5 \pm 1.0 \mathrm{~dB}$, which is comparable to conventional power divider/combiner on PCB. Isolation characteristic shows a 
value of $-16 \mathrm{~dB}$ at $10 \mathrm{GHz}$, and we can observe isolation characteristics higher than $-11 \mathrm{~dB}$ in the range of 1 to $25 \mathrm{GHz}$, which is sufficient for a dividing/combining of power.

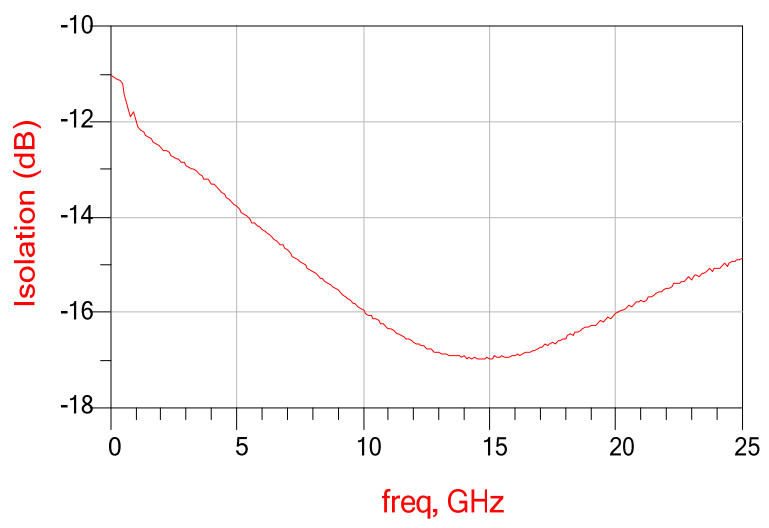

Fig. 8. Measured isolation characteristics of the 1-4 power divider/combiner employing PAGS

\section{CONCLUSIONS}

In this work, for application to a long distance wireless power transmission, we fabricated a highly miniaturized 1-4 power divider/combiner employing periodic structure on semiconducting silicon substrate. The 1-4 divider/combiner showed good RF performances from 1 to $25 \mathrm{GHz}$, and its size was $1.08 \times 0.63 \mathrm{~mm}^{2}$, which is $0.14 \%$ of the size of the one fabricated on PCB.

\section{ACKNOWLEDGMENT}

This research was supported by the MKE(The Ministry of Knowledge Economy), Korea, under the ITRC(Information Technology Research Center) support program supervised by the NIPA (National IT Industry Promotion Agency) (NIPA-2012-H0301-12-2009). This research was also supported by Basic Science Research Program through the National Research Foundation of Korea (NRF) funded by the Ministry of Education, Science and Technology (2010-0007452).

\section{REFERENCES}

[1] W. C. Brown, "The History of Power Transmission by Radio Waves," IEEE Trans. on Microwave Theory and Tech., vol. 32, pp. 1230- 1242 September 1984.

[2] J. D. L. S. Luk, A. Celeste, P. Romanacce, L. C. K. Sang, and J. C. Gatina, "Point-To-Point Wireless Power Transportation In Reunion Island,” in Proc. of 48th International Astronautical Congress, Turin, Italy, pp. 6-10 October, 1997

[3] Space Solar Energy Initiative. Space Island Group. [Online]. Available: http://www.spaceislandgroup.com/solarspace.html.

[4] Wireless Power Transmission for Solar Power Satellite (SPS) (Second Draft by N. Shinohara), Space Solar Power Workshop, Georgia Institute of Technology.

[5] M. Zargari and D. Su, "Challenges in designing CMOS wireless systems-on-a-chip,” IEICE Trnas. Electron, 2007, E90-C, pp. $1142-1148$.

[6] E. J. Wilkinson, “An N-way hybrid power divider," IRE trans, Microwave Theory Tech., vol. 8, pp. 116-118, Jun 1960.

[7] R. Dionisio and G. Andriolo, "High power X-band helix TWT for airbone radar applications," IEEE International Vacuum Electronics Conf., pp. 357-358, April, 2002.

[8] A. Kawano, N. Adachi, Y. Tateno, S. Mizuno, N. Ui, J. Nikaido, and S. Sano, "High-efficiency and wide-band single-ended 200W GaN HEMT power amplifier for $2.1 \mathrm{GHz}$ W-CDMA base station application,” Asia Pacific Conference Proceedings 2005 (APMC 2005), vol. 3, 2005.
[9] K. Kanto, A. Satomi, Y. Asahi, Y. Kashiwabara, K. Matsushita, and K. Takagi, "An X-band 250W Solid-state Power Amplifier using GaN Power HEMTs,” IEEE Radio and Wireless Symposium, pp. 77-80, 2008

[10] Y. Yun, M. Nishijima, M. Katsuno, H. Ishida, K. Minagawa, T. Nobusada, and T. Tanaka, "A Fully-Integrated Broadband Amplifier MMIC Employing a Novel Chip Size Package,” IEEE Trans. Microwave Theory Tech., vol. 50, pp. 2930-2937, Dec. 2002.

[11] Y. Itoh, M. Nii, N. Takeuchi, Y. Tsukahara, and H.Kurebayashi, "MMIC/Super-MIC/MIC-Combined C- to Ku-Band 2W Balanced Amplifier Multi-Chip Module,” IEICE Trans. Electron., vol. E80-C, no. 6, pp. 757-762, June 1997.

[12] D. Staiman and M. Breese, "200 Watt Solid State UHF Amplifier," Microwave Symposium, 1969 G-MTT International, pp. 445-449, May, 1969

[13] M. S. Kim, C. S. Lee, S. R. Lee, and Y. C. Rhee, "Implementation of the 200-Watts SSPA for X-band Pulse Compression Solid State Radar," The Institute of Electronics Engineers of Korea, vol.46, Chap.TC, no. 12, pp. 1126-1133, Dec.2009.

[14] Y. Yun, Y. B. Park, S. K. Kang, I. H. Kang, J. W. Jung, and K. H. Park, "Miniaturized On-chip Branch-line Coupler Employing Periodically Arrayed Grounded-strip Structure for Application to Silicon RFIC," Microwave Journal, vol. 52, no. 12, 2009, pp. 90-98.

[15] J. G. Ju, Y. Yun, Y. B. Park, and S. Y. Kang, “An Ultra-compact On-chip RF Divider Circuit Employing Periodically Arrayed Ground Strip Structure on RFIC," Microwave Journal, Accepted for publication.

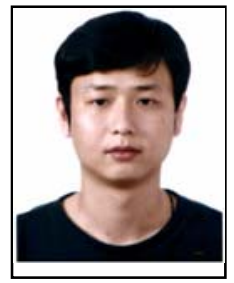

Jeong-Gab Ju received the B.S. degree in Radio Communication and Engineering from Korea Maritime University, 2010, respectively, and is currently working toward the M.S. degree at Korea Maritime University. Currently, he is working at the HANYANG INC., Daejeon, Korea.

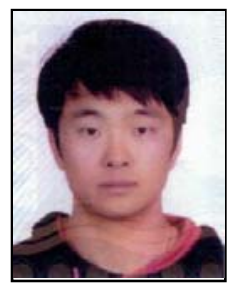

Jang-Hyeon Jeong received the B.S. degree in Radio Communication and Engineering from Korea Maritime University, 2010, respectively, and is currently working toward the M.S. degree at Korea Maritime University. Currently, he is studying at Korea Maritime University as Ph. D. course.

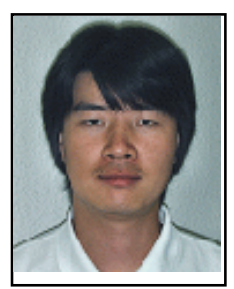

Young-Bae Park received the B.S., M.S. and Ph. D. degree in Radio Sciences and Engineering from Korea Maritime University, 2005, 2007and 2011, respectively. Currently, he is working at INTECH INC., Incheon, Korea.

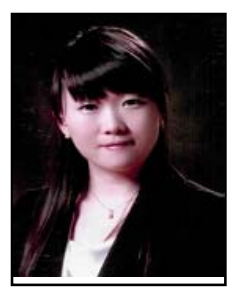

Bo-Ra Jung received the B.S. and M.S. degree in Radio Sciences and Engineering from Korea Maritime University, 2009 and 2011, respectively. Currently, she is working at INTECH INC., Incheon, Korea.

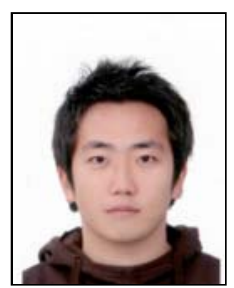
Communication and Engineering from Korea Maritime University, 2011, respectively, and is currently working toward the M.S. degree at Korea Maritime University.
Eui-Hoon Jang received the B.S. degree in Radio 


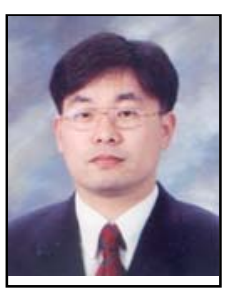

Suk-Youb Kang received the B.E. degree in electronics engineering from the University of Inchon, Incheon, Korea, in 1997, and the M.S and $\mathrm{Ph} . \mathrm{D}$. degrees in electronics engineering from the Inha University, Incheon, Korea, in 1999 and 2005, respectively. From 2006 to 2010, he worked as a research professor at the department of Radio Sciences and Engineering, Korea Maritime University, in Busan, Korea. Since 2001, he has been held position of representative director of INTECH Inc. and head of Electronics and Telecommunications Research Institute attached to there. His research interests include design and fabrication for microwave/wireless communication systems, and various antennas.

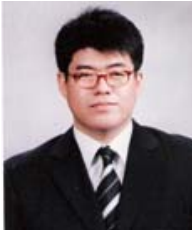

Young Yun received the B.S. degree in electronic engineering from Yonsei University, Seoul, Korea in 1993, and the M.S. degree in electrical and electronic engineering from Pohang University of Science and Technology, Pohang, Korea in 1995, and Ph.D. in electrical engineering from Osaka University, Osaka, Japan in 1999, respectively. From 1999 to 2003, he worked as an engineer in Matsushita Electric Industrial Company Ltd. (Panasonic), Osaka, Japan, where he has been engaged in the research and development of monolithic microwave ICs (MMICs) for wireless communications. In 2003, he joined Dept. of Radio Sciences and Engineering, Korea Maritime University, in Busan, Korea. Now he is currently a professor, and his research interests include design and measurement for RF/microwave and millimeter-wave IC, and flexible IC. Since 2008, he has served as an Associate Editor of IEICE (The Institute of Electronics, Information and Communication Engineers) in Japan, and an Editor of the Korean Society of Marine Engineering in Korea. He is the author and co-author of over 110 international journals and 15 patent pending in RF/microwave device and IC. 